\title{
Effect of coherent noise on single-station direction of arrival estimation
}

\section{Journal Article}

\section{Author(s):}

Greenhalgh, Stewart A.; Zhou, Bing; Rutty, Matthew

Publication date:

2008

Permanent link:

https://doi.org/10.3929/ethz-b-000011467

\section{Rights / license:}

In Copyright - Non-Commercial Use Permitted

\section{Originally published in:}

Journal of Seismology 12(3), https://doi.org/10.1007/s10950-007-9085-8 


\title{
Effect of coherent noise on single-station direction of arrival estimation
}

\author{
Stewart Alan Greenhalgh • Bing Zhou • \\ Matthew Rutty
}

Received: 23 September 2005 / Accepted: 30 November 2007 / Published online: 18 January 2008

(C) Springer Science + Business Media B.V. 2007

\begin{abstract}
Polarization analysis of multi-component seismic data is used in both exploration seismology and earthquake seismology. In single-station polarization processing, it is generally assumed that any noise present in the window of analysis is incoherent, i.e., does not correlate between components. This assumption is often violated in practice because several overlapping seismic events may be present in the data. The additional arrival(s) to that of interest can be viewed as coherent noise. This paper quantifies the error because of coherent noise interference. We first give a general theoretical analysis of the problem. A simple mathematical wavelet is then used to obtain a closed-form solution to the principal direction estimated for a transient incident signal superposed with a time-shifted, unequal amplitude version of itself, arriving at an arbitrary angle to the first wavelet. The effects of relative amplitude, arrival angle, and the time delay of the two wavelets on directional estimates are investigated. Even for small
\end{abstract}

S. A. Greenhalgh $(\bowtie) \cdot$ B. Zhou

Department of Physics, University of Adelaide, Adelaide, South Australia 5005, Australia

e-mail: greenhalgh@aug.ig.erdw.ethz.ch

S. A. Greenhalgh

Institute of Geophysics,

ETH Zürich 8093 Hönggerberg, Switzerland

M. Rutty

Petrosys Pty Ltd,

Kent Town, South Australia 5067, Australia differences in angle of arrival, there may be significant error $\left(>10^{\circ}\right)$ in the azimuth estimate.

Keywords Coherent noise $\cdot$ Seismic direction finding · Triaxial station

\section{Introduction}

The arrival direction of a seismic event, in terms of azimuth and inclination, can be estimated directly from measurements at a single triaxial station. Such direction finding is important in earthquake seismology in connection with hypocenter location and arrival identification, and in seismic exploration for imaging of reflectors and directional filtering. The problem has been tackled by a number of researchers (Flinn 1965; Montalbetti and Kanasewich 1970; Vidale 1986; Magotra et al. 1987; Cichowicz et al. 1988; Roberts et al. 1989; Jurkevics 1988; Jackson et al. 1991, 1999; Wagner and Owens 1991; Cho and Spencer 1992; Perelberg and Hornbostel 1994; Lilly and Park 1995; Ananat and Dowla 1997; Claassen 2000; Richwalshi et al. 2001; Greenhalgh et al. 2005). There are cases in which the angular resolution obtained by triaxial polarization analysis is better than that obtained by beam-forming with an extended array (Suteau-Hensen 1990; Gal'perin 1983; Ruud et al. 1988; Harris 1990; Bataille and Chiu 1991; Jarpe and Dowla 1991). For example, a single triaxial sensor has superior direction finding capability to a 
60-channel, 30-wavelength-long linear array when the source lies on boresite (perpendicular to the array). For an endfire configuration (e.g., a vertical seismic profiling), the array would need to be 1,700 wavelengths long to achieve comparable acuity. This is important for it is sometimes physically impossible to create a large receiving array of this aperture.

While there exist many large networks of seismic stations (global and regional) to accurately determine hypocenters of earthquakes, single-station earthquake location is still of interest for locating small magnitude local events detected by a single station. With isolated earthquake observatories or small sparse arrays, there is no alternative in locating events but to work with three-component seismograms. In these situations, one depends totally on understanding the interrelationships between the triaxial records forming a seismic wavetrain. The importance of estimating errors in arrival direction is related to improving location techniques and improving error estimates. This is true not only for single stations but also for arrays and networks of stations.

There are several approaches to estimating the direction of arrival of a seismic event from vector particle motion records at a single station, as revealed in the references cited above. Nearly all methods start by using the multi-component data to form a covariance matrix (or its equivalents: the crossspectral matrix in the frequency domain and the coherency matrix when using the complex analytic signal). This matrix is constructed by forming the outer product of the multi-component data vector with itself at each data point within a nominated data window. This produces individual matrices for each data point, which are then averaged over the data window. The form of the averaged matrix indicates any linear dependencies between the data components within the averaging window. The eigenvectors of the matrix are a primary source of directional information. Its eigenvalues measure the amplitude and scatter of the data vectors.

Theoretical studies by Harris (1990) and Greenhalgh et al. (2005), and numerical/empirical studies by Jarpe and Dowla (1991), Walck and Chael (1991), Hearn and Hendrick (1998), Knowlton and Spencer (1996), Claassen (2000), Richwalshi et al. (2001), and others suggest that three-component stations exhibit a gradual decay of performance with respect to azimuth and inclination estimation as the signal-to-noise ratio
(SNR) decreases. The error in arrival direction seems to vary inversely with the SNR, being around $3^{\circ}$ at a SNR of 1 and dropping to $0.5^{\circ}$ at a SNR of 8 . However, systematic and potentially large errors in estimated arrival direction arise if the noise is coherent between the recording channels, if seismic modes are incorrectly identified, and if multipath arrivals share the same averaging window (Rutty and Greenhalgh 1993, 1999; Wagner 1996; Claassen 2000). The multi-path problem becomes important in the presence of near-source and near-receiver scatterers or significant inhomogeneities along the propagation path. The scattered phases and other events on the seismic section may overlap and, hence, interfere with each other. This is the reason that polarization processing performed on real seismic data has been more successful when applied to the first P-wave arrivals, which suffer less from interference than do the later arrivals. Errors in system calibration (e.g., interchannel gain variations), poor sensor coupling to the ground (which can change the relative amplitudes on the different components, as well produce phase distortion), and refractions at local interfaces also contribute systematic direction finding errors. Accurate station calibration is, therefore, essential. A rigorous treatment of the multipath problem and how to handle coherent noise really requires an array of three component sensors to exploit time differences (moveout), as well as polarization properties of the wavefield. Multichannel vector processing analyses have been given by Greenhalgh et al. (1992), Wagner (1996), and Claassen (2000) but are beyond the scope of the present study. Furthermore, they require that the array stations are at a spacing significantly less than the coherence aperture of the wavefield, which cannot always be assured. A single-station approach is often all that can be done.

In this paper, we quantify the direction of arrival error because of coherent noise interference at a single triaxial station. We begin by looking at the problem of polarization direction estimation in the presence of purely random noise. Next, we give a general triaxial analysis of the coherent noise problem for unequal wavelets (arbitrary directions and pulse shapes) that provides insight to the direction finding issue. We then use a simple mathematical wavelet to obtain a closed form solution to the two-component (biaxial) station problem for determining the dominant eigen- 
vector for the case of a transient incident signal superposed with a time-shifted, unequal amplitude version of itself, arriving at an arbitrary angle to the first wavelet. The effects of relative amplitude, arrival angle, and the time delay of the two wavelets on directional estimates are investigated. Graphs are presented that characterize the magnitude of the error in practice. The only solution to the coherent noise problem is to utilize additional triaxial stations in the form of a vector interferometer (see Rutty and Greenhalgh 1999).

\section{Single-station polarization directional estimates in random noise}

The basic theory of polarization processing is based on a model, assuming only a single arrival, $f_{1}(t)$, plus random noise, $n(t)$, within the selected window of data under examination. This model is stated mathematically as

$\mathbf{r}(t)=\mathrm{f}_{1}(t) \cdot \hat{\mathbf{c}}+\mathbf{n}(t)$

where $c$ is the polarization direction and may be complex. Processing relies on the linear dependence of the signal and the incoherence of the noise over the window of data in forming the covariance matrix.

The polarization direction estimated by the principal eigenvector of the covariance matrix, formed from a three-component data set, is an unbiased estimate of the polarization direction of a rectilinear event in truly random noise, whatever the amplitude of the noise. This is shown below.

Without loss of generality, consider a rectilinear signal, $f$, arriving at a detecting station with direction, $\boldsymbol{c}$, given by the direction ratios $(1: 1: 1)$. This is a general case for a single signal at an arbitrary direction, as the co-ordinate axes may be rotated such that the signal direction lies along $c$. Any covariance matrix, $R$, formed from the noise-free traces will be of the form

$\mathbf{R}=\sigma c c^{*}=\sigma\left[\begin{array}{lll}1 & 1 & 1 \\ 1 & 1 & 1 \\ 1 & 1 & 1\end{array}\right]$

where $\sigma$ is the mean signal energy. The covariance matrix of any random noise to be superposed on the signal will be a diagonal matrix (Born and Wolf 1975; Kanasewich 1981) of the form

$\mathbf{N}=\left[\begin{array}{lll}k & 0 & 0 \\ 0 & k & 0 \\ 0 & 0 & k\end{array}\right]$

The combined signal and noise may, therefore, be described by

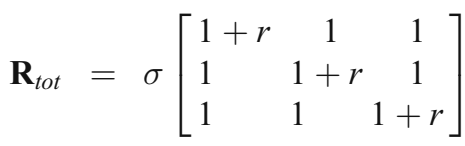

where $r$ is the noise-to-signal ratio $(k / \sigma)$.

This matrix has characteristic equation

$3(r-\lambda)^{2}+(r-\lambda)^{3}=0$

which implies eigenvalues $\lambda$ equal to $r, r$ and $r+3$. Since $r>0$, the principal eigenvalue is clearly $r+3$. By substitution in the eigenequation,

$\mathbf{R}_{t o t} \mathbf{u}=(r+3) \mathbf{u}$

its corresponding eigenvector, $u$, is trivially shown to be

$\boldsymbol{u}=\frac{1}{\sqrt{3}}\left(\begin{array}{l}1 \\ 1 \\ 1\end{array}\right)$

Thus, the polarization direction is correctly estimated in totally random noise, irrespective of the amplitude of the noise-to-signal ratio $r$. This important property is one of the major reasons for the success of single-station polarization analysis of such data. The major concern with this type of analysis is how well the data fit the assumption of random noise.

Consider now the effect of random noise having different amplitudes on each channel. This may be illustrated analytically by a simple two-dimensional example. The covariance matrix of a polarized event arriving in $2 \mathrm{D}$ space with direction ratios $(1: 1)$ and relative random noise levels, $\delta$ and $\varepsilon$, on each component may be represented as

$R=\left[\begin{array}{ll}1+\delta & 1 \\ 1 & 1+\varepsilon\end{array}\right]$ 
This has principal eigenvalue

$\lambda_{1}=1+\frac{\delta+\varepsilon}{2}+\frac{\sqrt{(\delta-\varepsilon)^{2}+4}}{2}$

with corresponding non-normalized eigenvector:

$$
\left(\varepsilon-\delta+\sqrt{(\varepsilon-\delta)^{2}+4}\right)
$$

The estimated polarization direction will, therefore, only be correct $(2,2)$ if the noise amplitude is equal on each channel $(\varepsilon=\delta)$, a point often ignored in polarization analysis.

We showed above on purely theoretical grounds that an eigendecomposition of the covariance matrix should succeed in finding the direction of any signal in purely random noise no matter how high the levels of random noise. In practice, this is not the case. The concept of noise being totally random is purely a statistical notion. Noise added to the signal is less likely to cancel when averaged over a small time window than it would using a larger one. We expect the error to be inversely proportional to the timebandwidth product (window length). It is the variance of the estimated direction that is determined by the SNR. If an experiment could be repeated an infinite number of times or if the time window used in forming the covariance matrix were infinitely long, then there would be no error in the polarization direction estimate no matter how small the SNR is. This is not possible in practice. The computed azimuth will have an error related to the variance of the noise distribution. This is the reason why polarization processing is generally unsuccessful with single-mode data with a SNR less than 1.

\section{Interfering events and coherent noise}

When two purely polarized events overlap (and, therefore, interfere) within the window used for polarization analysis, a composite nonpolarized particle motion is produced. This may be visualized by examining the hodograms of three windows of a synthetic seismogram (Fig. 1). The hodogram is a crossplot of the various two components of motion (e.g., $\mathrm{X}-\mathrm{Y}, \mathrm{X}-\mathrm{Z}, \mathrm{Y}-\mathrm{Z}$ ) over a time window to gain an impression of the $3 \mathrm{D}$ particle displacement as a function of time. In window a, there is no significantly polarized signal, producing a scattered hodogram. In window $b$, there is a strong linear event, indicated by the corresponding hodograms. In window c, two linearly polarized events overlap, producing a composite signal producing complicated hodograms.
Fig. 1 A three-component trace illustrating hodogram for a wave, $\mathbf{b}$ a single rectilinearly polarized event, c overlapping rectilinear events

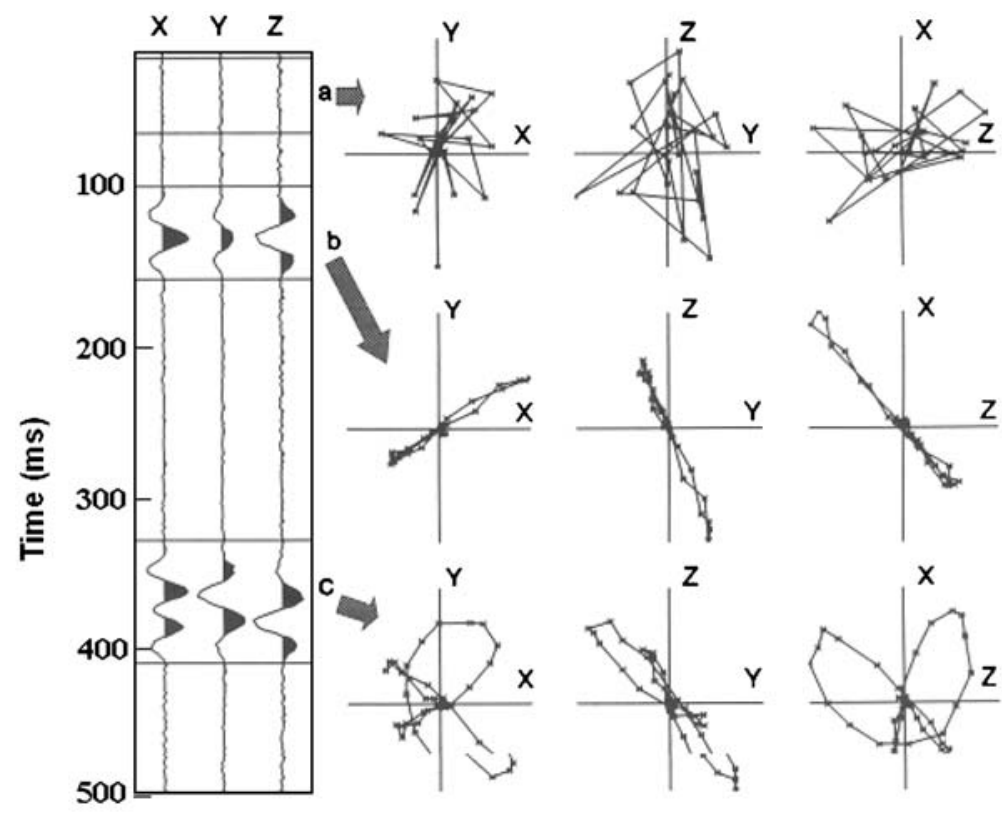


If polarization analysis is applied to data with significant interference such as in case c above, then in general, none of the principal directions obtained from an eigenanalysis will then point along the direction of the individual event directions. All that is then known is that the original direction vectors of the two signals both lie in the plane spanned by the two principal directions of the covariance matrix. Any signal interfering with the signal of interest is then a form of coherent noise and is a major problem in a standard single station covariance analysis.

\section{General triaxial analysis}

Consider a model involving two superposed signals $f_{1}(t)$ and $f_{2}(t)$, arriving at vector directions given by the direction cosines $\underline{c}_{1}$ and $\underline{c}_{2}$ :

$\underline{r}(t)=\underline{c}_{1} f_{1}(t)+\underline{c}_{2} f_{2}(t)$

where $r$ is the composite signal and tenotes the time dependency of the unequal wavelets $f_{1}(t)$ and $f_{2}(t)$. If the signals are defined on the interval $[O, L]$, then the sample covariance matrix is

$\underline{R}=\underline{c}\left[\begin{array}{cc}\sigma_{1}^{2} & \sigma_{1} \sigma_{2} \gamma \\ \sigma_{1} \sigma_{2} \gamma & \sigma_{2}^{2}\end{array}\right] \underline{c}^{T}$

where the signal energies are

$\sigma_{i}^{2}=\int_{0}^{L} f_{i}^{2}(t) d t \quad \mathrm{i}=1,2$

and the correlation coefficient or coherence function between the two signals is

$\gamma=\int_{0}^{L} \frac{f_{1}(t) f_{2}(t) d t}{\sigma_{1} \sigma_{2}}$

and the matrix $\mathbf{c}$ has as its two column vectors, $\boldsymbol{c}_{1}$ and $\boldsymbol{c}_{2}$. The covariance matrix can be decomposed into the form

$$
\underline{R}=\left[\begin{array}{ll}
\sigma_{1} \underline{c}_{1} & \sigma_{2} \underline{c}_{2}
\end{array}\right]\left[\begin{array}{l}
1 \gamma \\
\gamma 1
\end{array}\right]\left[\begin{array}{ll}
\sigma_{1} & \frac{c_{1}^{T}}{\sigma_{2}} \\
c_{2}^{T}
\end{array}\right]
$$

By means of the decomposition

$$
\left[\begin{array}{ll}
1 & \gamma \\
\gamma & 1
\end{array}\right]=\frac{1}{2}\left[\begin{array}{cc}
1 & 1 \\
1 & -1
\end{array}\right]\left[\begin{array}{cc}
1+\gamma & 0 \\
0 & 1-\gamma
\end{array}\right]\left[\begin{array}{cc}
1 & 1 \\
1 & -1
\end{array}\right]
$$

the covariance matrix takes the following form:

$\underline{R}=\left[\begin{array}{ll}\sigma_{1} \underline{\underline{c}}_{1}+\sigma_{2} c_{2} & \sigma_{1} \underline{c}_{1}-\sigma_{2} \underline{\underline{c}}_{2}\end{array}\right]\left[\begin{array}{cc}\frac{1+\gamma}{2} & 0 \\ 0 & \frac{1-\gamma}{2}\end{array}\right]\left[\begin{array}{l}\left(\sigma_{2} \underline{c}_{2}+\sigma_{1} \underline{c}_{1}\right)^{T} \\ \left(\sigma_{1} \underline{\underline{c}}_{1}-\sigma_{2} \underline{\underline{c}}_{2}\right)^{T}\end{array}\right]$

We expect the error in estimated azimuth (so-called bearing swing — see Gething 1991) to be maximal when the two orthogonal arrivals are $180^{\circ}$ out of phase. In this case, the correlation coefficient is $\gamma=-1$ and

$\underline{R}=\left(\sigma_{1} \underline{c}_{1}-\sigma_{2} \underline{c}_{2}\right)\left(\sigma_{1} \underline{c}_{1}-\sigma_{2} \underline{c}_{2}\right)^{T}$

For signals of equal amplitude $\left(\sigma_{1}=\sigma_{2}\right)$, the principal eigenvector is $\boldsymbol{c}_{1}-\boldsymbol{c}_{2}$. As the incident waves are orthogonal, the azimuth (bearing) swings 45 or $135^{\circ}$ from the two incident wavefield directions.

For the case of two in-phase arrivals $(\gamma=1)$ the covariance matrix is

$\underline{R}=\left(\sigma_{1} \underline{c}_{1}+\sigma_{2} \underline{c}_{2}\right)\left(\sigma_{1} \underline{c}_{1}+\sigma_{2} \underline{c}_{2}\right)^{T}$

and a similar result obtains for the computed azimuth.

\section{A simple mathematical model for biaxial data}

To quantify the errors on azimuth estimation, we will now consider a very simplified mathematical model involving two-component (biaxial) data in which the same transient wave defines each pulse. The wavelet is defined by

$$
f(t)=\frac{4 t}{L}\left(1-\frac{t}{L}\right) \quad t \in[o, L]
$$

where $L$ is a constant defining the wavelet period or duration. The wavelet and a delayed version of itself are shown in Fig. 2. It is taken to be unipolar and of positive sign for ease of treatment. We are not supposing that actual seismic signals are of such simple form. They are obviously much more complicated. Nevertheless, the simplified model will allow us to illustrate the multipathing effect and complement the more general treatment given earlier. 


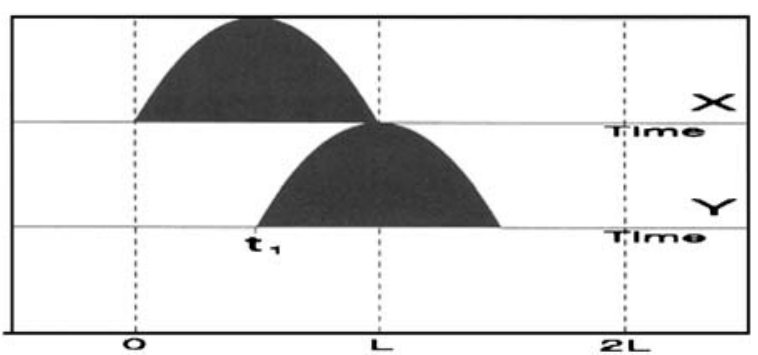

Fig. 2 The simple transient wavelets defined in Eq. 21 with $\theta>$ 0 used for a two-dimensional analytical test. Two correlating orthogonal events arrive on $X$ and $Y$ components with a time delay $t_{1}$

The effect of interference of two such wavelets may be investigated by considering the two component data $\underline{r}=(X, Y)$ defined below:

$$
\begin{gathered}
X=a_{1} f(t) \cos \theta_{1}+a_{2} f\left(t-t_{1}\right) \cos \theta_{2} \\
Y=a_{1} f(t) \sin \theta_{1}+a_{2} f\left(t-t_{1}\right) \sin \theta_{2}
\end{gathered}
$$

These represent two similar signals $\left(f_{1}=a_{1} f(t), f_{2}=\right.$ $\left.a_{2} f\left(t-t_{1}\right)\right)$ with respective amplitudes $a_{1}$ and $a_{2}$, angles of arrival around $\theta_{1}$ and $\theta_{2}$, and times of arrival 0 and $t_{1}$. Note that in the previous analysis (see Eq. 1),

$\underline{c}_{1}=\left(\cos \theta_{1}, \sin \theta_{1}\right)$

$\underline{c}_{2}=\left(\cos \theta_{2}, \sin \theta_{2}\right)$

Without loss of generality, we may allow the first event to arrive along the $x$-axis (i.e., $\theta_{1}=0$ as plotted in Fig. 2). We can now examine the covariance matrix formed from such a data set, and note its dependence on relative amplitudes, time delays, and angle between arrival directions of the two wavelets. The closed form of the principal eigenvector of this matrix yields the apparent direction of arrival. By comparing with the true (known) direction of the event in question, we gain insight into how interference by another arrival affects directional errors even in an environment totally free of random noise.

The covariance matrix (Eq. 12) formed from the model in Eq. 21, with $\theta_{1}$ set to zero and limits of integration from 0 to $L$, is

$R=\frac{16}{30} L\left[\begin{array}{lc}a_{1}^{2}+2 a_{1} a_{2} \cos \left(\theta_{2}\right) I_{x y}+a_{2}^{2} \cos ^{2}\left(\theta_{2}\right) I_{y y} & a_{1} a_{2} \sin \left(\theta_{2}\right) I_{x y}+a_{2}^{2} \cos \left(\theta_{2}\right) \sin \left(\theta_{2}\right) I_{y y} \\ a_{1} a_{2} \sin \left(\theta_{2}\right) I_{x y}+a_{2}^{2} \cos \left(\theta_{2}\right) \sin \left(\theta_{2}\right) I_{y y} & a_{2}^{2} \sin ^{2}\left(\theta_{2}\right) I_{y y}\end{array}\right]$

where

$$
\begin{aligned}
I_{x y}= & \int_{0}^{L} f \cdot f\left(t-t_{1}\right) d t=1-u-5 u^{2}+5 u^{3} \\
I_{y y}= & \int_{0}^{L} f^{2}\left(t-t_{1}\right) d t=1-10 u^{3}+15 u^{4} \\
& -6 u^{5}
\end{aligned}
$$

and $u=\frac{t_{1}}{L}$.

The eigenvalues, $\lambda_{1}$ and $\lambda_{2}$, of a $2 \times 2$ symmetric matrix:

$$
M=\left[\begin{array}{ll}
A & B \\
B & D
\end{array}\right]
$$

may be written in closed form as

$\lambda_{i}=\frac{(A+D)}{2} \pm \sqrt{\frac{(A-D)^{2}+4 B^{2}}{2}} \quad i=1,2$
The eigenvector, $\boldsymbol{\nu}_{\mathbf{1}}$, corresponding to the principal eigenvalue is given in nonnormalized form as

$\boldsymbol{v}_{\mathbf{1}}=\left[\begin{array}{c}2 B \\ D-A+\sqrt{(A-D)^{2}+4 B^{2}}\end{array}\right]$

or in normalized form as

$\boldsymbol{v}_{\mathbf{1}}=\left[\begin{array}{c}\cos \psi \\ \sin \psi\end{array}\right]$

where $\psi$ is the apparent polarization direction and

$\tan \psi=\frac{D-A+\sqrt{(A-D)^{2}+4 B^{2}}}{2 B}$

An eigenanalysis can, therefore, be directly performed on the covariance matrix given in Eq. 23 and the variation of the principal eigenvector, $\nu_{\mathbf{1}}$, observed. The apparent azimuth of arrival given by Eq. 28 depends on the quantities $A, B, C$, and $D$, 
which are functions of the amplitude ratio of the two interfering arrivals $a_{1} / a_{2}$, the actual arrival direction $\theta_{2}$, and the time delay $t_{1}$ for the two signals.

\section{Azimuth error}

Figure 3 shows the variation of computed direction (apparent azimuth) against delay time of the second event, expressed as a fraction of a period. The amplitudes of the two events were made equal $\left(a_{1}=\right.$ $a_{2}$ ), and their true arrival directions were orthogonal $\left(\theta_{1}=0^{\circ}, \theta_{2}=90^{\circ}\right)$. The covariance matrix is computed over a fixed time window length $L$. When the events arrive simultaneously $\left(t_{1}=u=0\right)$, the two components of data have equal amplitude and phase with the composite motion being rectilinear. The apparent polarization direction is then $45^{\circ}$ (intermediate between the two wave directions).

When there is no interference from the second event (no overlap, i.e., $t_{1}=L$ or $u=1$ ), only the first event contributes to the covariance matrix, and again, the rectilinear motion is correctly detected with a correctly estimated arrival direction of $0^{\circ}$. However, at other time delays, there is significant variation from either of the known signal arrival directions or their average, as shown in Fig. 3. The most significant

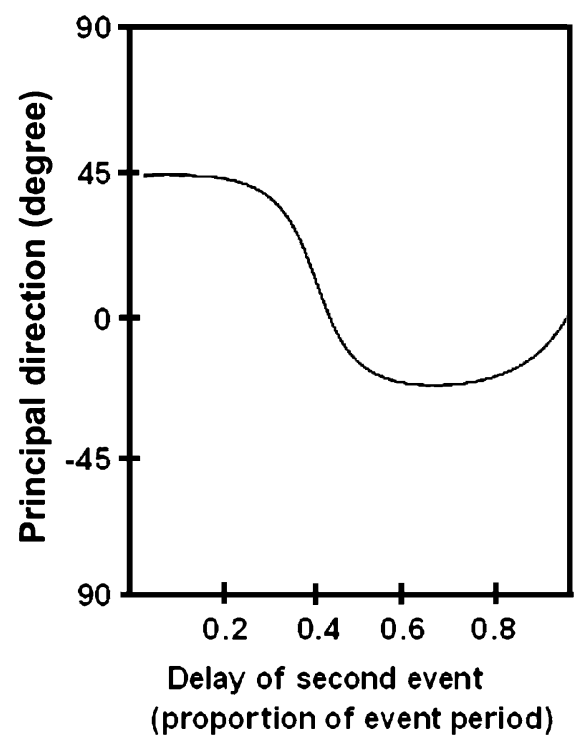

Fig. 3 Single-station direction finding in coherent noise. The estimated direction of arrival for the transient events in Fig. 2 is plotted as their delay time varies. The two events have equal amplitude and arrive at an angular separation of $90^{\circ}$. The true arrival direction of event 1 is $0^{\circ}$

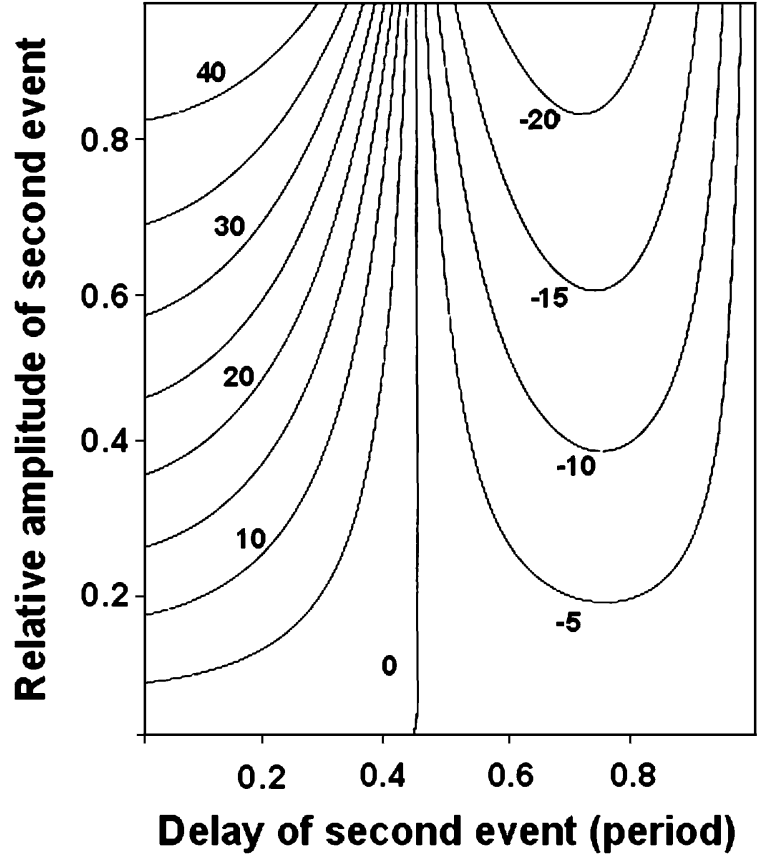

Fig. 4 Bearing variation with relative amplitude for two wavelets arriving at an angular separation of $90^{\circ}$. The bearing swing is most apparent (i.e., contours are closest) for equal amplitude event. The azimuth error is severe at small delays

variation occurs for time delays of around $0.4-0.5$ of the duration of the unipolar pulse.

This effect, known as bearing swing, has been studied for monochromatic electromagnetic signals (Gething 1991) and appears to have maximum effect

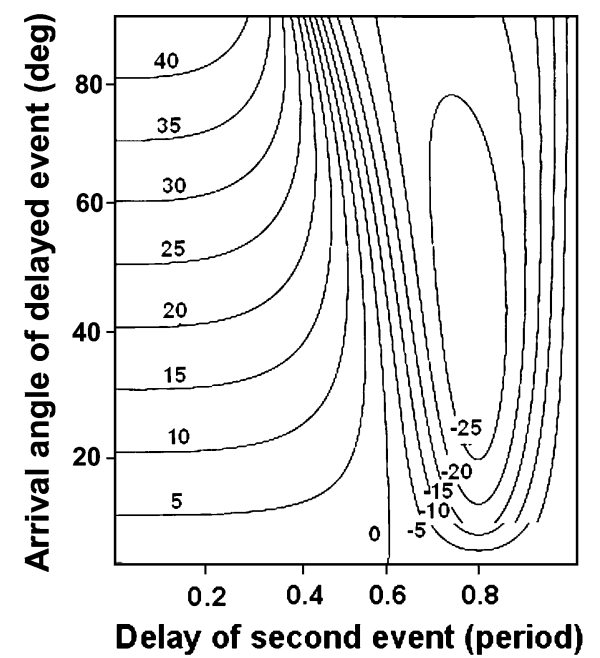

Fig. 5 The variation of estimated bearing with difference in angle of arrival for two equal amplitude events. As the difference in arrival angle increases, the azimuth error worsens. The maximum gradient occurs at a delay of about half a period 
when the phase difference between interfering waves is $180^{\circ}$. Of course, for such sinusoidal signals, there are positive and negative amplitudes, and interference can be destructive or constructive. Similarly, when polychromatic transient waves are being analyzed, there are several oscillating cycles of the waveform and one might expect maximum effect when the interfering waves have a phase shift of $180^{\circ}$. This corresponds to a time shift of about half the dominant period $(\approx 0.5 \mathrm{~L})$. The result displayed in Fig. 3 is, therefore, consistent with this observation.

If the ratio of the amplitudes of the wavelets, $a_{1} / a_{2}$, is allowed to vary, the bearing swing will change. Figure 4 is a contour plot of apparent polarization direction (i.e., principal eigenvector) as a function of both time delay (linear phase shift) and relative amplitude. Again, the arrival directions of the events are separated by $90^{\circ}$. The variation of the calculated bearing with time delay has a similar shape for all values of the relative amplitude but is less pronounced for small relative amplitude. This is because of the stronger signal dominating the data. The higher the amplitude ratio, the more closely the calculated angle of arrival will track the true arrival direction of the stronger signal, and the less the influence of the weaker the signal on the apparent polarization direction.

If the angle of arrival of the delayed event is allowed to vary but the amplitude ratio of the two signals is kept constant, the plot shown in Fig. 5 is obtained. By contrast to Fig. 4, the delay giving steepest gradient is no longer constant. As the angle between arrival directions increases from 0 to $90^{\circ}$, the time delay producing the greatest bearing swing moves from 0.6 to 0.4 of a period. The most extreme bearing swing is seen to occur for orthogonal events of equal amplitude; this case was shown in Fig. 3.

\section{Conclusions}

A major advantage of using three-component seismic stations is that directional information can be inferred from a single location in space. All single-station triaxial direction finding techniques search for pure modes of vibration and assume that the noise on the signal is random. This paper shows analytically that totally random noise cannot affect directional estimation in a single-station polarization analysis. However, the direction finding ability will be severely affected by any overlapping signals within the analysis window.

In this paper, we give a formal theoretical analysis of the coherent noise problem for two dissimilar wavelets arriving from different directions at a single triaxial station. Equations are derived that show the form of the covariance matrix. We then consider a particularly simple form of wavelet for the biaxial problem of azimuth estimation and give closed form expressions for the eigenvector corresponding to the dominant eigenvalue. This direction estimate is cast in terms of the amplitudes, arrival directions, and phase lags for the two interfering arrivals. The azimuth error is most severe when interfering events have approximately the same amplitude. The effect is also dependent upon the difference in polarization angle of the two signals and is strongest when they have orthogonal polarization. However, even for small differences in angle of arrival $\left(<5^{\circ}\right)$, there may be a significant $\left(>20^{\circ}\right)$ error in the direction estimate.

The direction estimate of interfering events not only has an error, but it also varies dramatically with relative time delay or phase difference. The estimated azimuth direction swings most from its true value for time delays of about $0.4-0.5$ of the dominant period between interfering events.

We have shown that, in the case of random noise, when the SNR is low or if there are multiple interfering arrivals (coherent noise), the single triaxial station approach to direction finding will not be of benefit. A multi-station approach, which allows separation of interfering events, is then imperative. It is the only way to effectively combat coherent noise and allow an accurate estimate of azimuth and inclination for a given seismic arrival.

Acknowledgment This research was supported by the Australian Research Council. We thank Dr. D. Harris who reviewed an earlier version of the manuscript and suggested the more general treatment.

\section{References}

Ananat KS, Dowla FU (1997) Wavelet transform methods for phase identification in three-component seismograms. Bull Seismol Soc Am 87:1598-1612

Bataille K, Chiu JM (1991) Polarization analysis of highfrequency, three-component seismic data. Bull Seismol Soc Am 81:622-642

Born M, Wolf E (1975) Principles of optics. Pergamon, New York 
Cichowicz AR, Green WE, van Zyl Brink A (1988) Coda polarization properties of high-frequency microseismic events. Bull Seismol Soc Am 78:1297-1318

Cho WH, Spencer TW (1992) Estimation of polarization and slowness in mixed wavefields. Geophysics 57:805814

Claassen JP (2000) Robust bearing estimates from three component stations. Pure Applied Geophys 158:349374

Flinn EA (1965) Signal analysis using rectilinearity and direction of particle motion. Proc IEEE 53:1874-1876

Gal'perin EI (1983) The polarization method of seismic exploration. Reidel, Amsterdam

Gething PJD (1991) Radio direction finding and superresolution, 2nd edn. Peregrinus, Stevenage, UK

Greenhalgh SA, Mason IM, Lucas E, Pant DR, Eames RT (1992) Controlled direction reception filtering of P- and Swaves in tau-p space. Geophys J Int 100:221-234

Greenhalgh SA, Mason IM, Zhou B (2005) An analytical treatment of single station triaxial seismic direction finding. J Geophys Eng 2:8-15

Harris DB (1990) Comparison of the directional estimation performance of high-frequency seismic arrays and threecomponent stations. Bull Seismol Soc Am 80:1951-1968

Hearn S, Hendrick N (1998) A review of single-station timedomain polarization analysis techniques. J Seism Explor 8:181-202

Jackson GM, Mason IM, Greenhalgh SA (1991) Principal component transforms of triaxial recordings by singular value decomposition. Geophysics 56:528-533

Jackson GM, Mason IM, Greenhalgh SA (1999) Single station triaxial seismic direction finding. In: Kirlin L, Done W (eds) Covariance analysis in geophysics. Society Exploration Geophysics, Tulsa, OK, pp 275-290

Jarpe S, Dowla F (1991) Performance of high-frequency threecomponent stations for azimuth estimation from regional seismic phases. Bull Seismol Soc Am 81:987-999

Jurkevics A (1988) Polarization analysis of three-component array data. Bull Seismol Soc Am 78:1725-1743

Kanasewich E (1981) Time sequence analysis in geophysics. University of Alberta Press, Alberta, Canada

Knowlton KB, Spencer TW (1996) Polarization measurement uncertainty on three-component VSPs. Geophysics 61: 594-599
Lilly JM, Park J (1995) Multiwavelet spectral and polarization analysis of seismic records. Geophys J Int 122:1001-1021

Magotra NN, Ahmed E Chael E (1987) Seismic event detection and source location using single-station (three-component) data. Bull Seismol Soc Am 77:958-971

Montalbetti JF, Kanasewich ER (1970) Enhancement of teleseismic body phases with polarization filter. Geophys J R Astron Soc 21:119-129

Perelberg AI, Hornbostel SC (1994) Applications of seismic polarization analysis. Geophysics 59:119-130

Richwalshi S, Roy-Chowdury K, Mondt JC (2001) Multicomponent wavefield separation applied to high resolution surface seismic data. J Appl Geophys 46:101-114

Roberts RG, Christofferson A, Cassidy F (1989) Real-time event detection, phase identification and source location estimates using single station three-component seismic data. Geophys J Int 97:471-480

Rutty MJ, Greenhalgh SA (1993) The correlation of seismic events on multicomponent data in the presence of coherent noise. Geophys J Int 113:343-358

Rutty MJ, Greenhalgh SA (1999) Correlation using triaxial data from multiple stations in the presence of coherent noise. In: Kirlin L, Done W (eds) Covariance analysis in geophysical data processing. Society of Exploration Geophysicists, Tulsa, pp 291-322

Ruud BO, Husebye ES, Ingate SF, Christoffersson A (1988) Event location at any distance using seismic data from a single, three-component station. Bull Seismol Soc Am 78:308-325

Suteau-Hensen A (1990) Estimating azimuth and slowness from three-component and array stations. Bull Seismol Soc Am 80:1987-1998

Vidale JE (1986) Complex polarization analysis of particle motion. Bull Seismol Soc Am 76:1393-1405

Wagner GS (1996) Resolving diversely polarized superimposed signals in three component seismic array data. Geophys Res Lett 23:1837-1840

Wagner GS, Owens TJ (1991) Broadband eigen-analysis for three-component seismic array data. IEEE Trans Signal Proc 43(7):1738-1741

Walck MC, Chael EP (1991) Optimal backazimuth estimation for three-component recordings of regional seismic events. Bull Seismol Soc Am 81:643-666 